\title{
Integrated Silicon Nitride Microdisk Lasers Based on Quantum Dots
}

\author{
Weiqiang Xie, ${ }^{1}$ Thilo Stöferle, ${ }^{2}$ Gabriele Rainò,${ }^{2}$ Tangi Aubert, ${ }^{3}$ Yunpeng Zhu, ${ }^{1}$ Rainer F. Mahrt, ${ }^{2}$ Edouard \\ Brainis, ${ }^{3}$ Zeger Hens, ${ }^{3}$ and Dries Van Thourhout ${ }^{1}$ \\ ${ }^{\text {I}}$ Photonics Research Group, Department of Information Technology (INTEC), Ghent University-IMEC, Gent B-9000, Belgium \\ ${ }^{2} I B M$ Research-Zurich, Säumerstrasse 4, 8803 Rüschlikon, Switzerland \\ ${ }^{3}$ Physics and Chemistry of Nanostructures, Ghent University, B-9000 Ghent, Belgium \\ weiqiang.xie@intec.ugent.be
}

\begin{abstract}
On-chip waveguide-coupled silicon nitride microdisk lasers are demonstrated using a $55 \mathrm{~nm} \mathrm{CdSe} / \mathrm{CdS}$ quantum dots film as gain material. The devices were fabricated with a hybrid integration technique able to embed quantum dots in SiN cavities. Lasing is achieved under optical pumping with a record low threshold of $26.8 \mu \mathrm{J} \cdot \mathrm{cm}^{-2}$ in a $7 \mu \mathrm{m}$-diameter disk.

OCIS codes: (130.0130) Integrated Optics; (140.0140) Lasers and Laser Optics; (160.4236) Nanomaterials
\end{abstract}

\section{Introduction}

Integrated photonics allows to miniaturize optical functions within wavelength-scale structures on a chip. Relying on mature CMOS manufacturing processes, it has become a promising platform both for cost-effective and highvolume applications such as optical communication, information processing or optical sensing as well as for fundamental optics research in various domains such as nonlinear optics, cavity quantum electrodynamics and quantum optics. In particular, the recently developed silicon nitride (SiN) integrated photonics platform, given its high optical index and broad optical transparency, allowing to operate over a wide range of wavelengths is currently attracting a lot of attention and a variety of building blocks including low-loss waveguides and high quality (Q) resonators have been demonstrated. Those are mostly limited to passive functions though and active devices, in particular integrated lasers, are still highly desirable.

In this work, we demonstrate integrated $\mathrm{SiN}$ microlasers by combining on-chip SiN resonators with semiconductor colloidal quantum dots (QDs) as the gain medium. Gain at low optical-pumping has been demonstrated in such QDs [1,2]. Moreover, the gain wavelength in QDs is easily adjustable exploiting sizedependent quantum confinement and correct material choice, perfectly matching the broadband transparency of SiN photonics. Our laser cavities consist of microdisks defined in a SiN/QD/SiN sandwich with the centrally embedded QD layer providing optical gain. For efficient light extraction, we designed a vertical waveguide-cavity coupling scheme. Optimally designed microdisks feature high Q (up to 6,000) whispering gallery modes (WGM) with a maximum optical confinement in the QD layer. The devices were realized using an effective integration technique able to handle solution-processible QDs within a top-down CMOS-like manufacturing flow. Lasing in the emission band of $\mathrm{CdSe} / \mathrm{CdS}$ QDs is demonstrated for differently sized disks under picosecond optical pumping through a pronounced power increase and reduced lifetime of the waveguide coupled emission concurring with a spectral narrowing and enhanced temporal coherence. Record-low lasing threshold of $26.8 \mu \mathrm{J} \cdot \mathrm{cm}^{-2}$ for $7 \mu \mathrm{m}$ disks has been achieved in these ultra-compact waveguide-coupled SiN-QD microdisks thus opening the prospect of an active $\mathrm{SiN}$ integrated photonics platform based on colloidal QDs.

\section{Design and Fabrication}

Fig. 1(a) depicts the schematics of the device configuration with a SiN-QD microdisk vertically coupled to a passive bus waveguide. The details of the design are similar to our previously reported results [3]. Following finitedifference time-domain simulations (Fig. 1b), a $7 \times 0.25 \mu \mathrm{m}$ SiN disk supports a fundamental transverse-electric (TE) WGM with a simulated Q factor of $5 \times 10^{4}$ at $630 \mathrm{~nm}$, which is the central emission wavelength of the QDs to be integrated. Importantly, the optical confinement is 2-3 times higher in the center of the disk as compared to its surface, implying the same optical enhancement factor for QDs embedded in the disk. The structure is made by first fabricating the passive $\mathrm{SiN}$ waveguide chip with planarized oxide cladding using a low-loss $(\sim 1 \mathrm{~dB} / \mathrm{cm}$ at $630 \mathrm{~nm})$ $\mathrm{SiN}$ fabrication platform [4]. Then a uniform SiN/QD/SiN layer stack is deposited and disks are defined through optimized deposition and dry etching processes. The embedded QDs are $\sim 9.1 \mathrm{~nm}$ 'flash' CdSe/CdS QDs with 3.6 $\mathrm{nm}$ core size [5]. Fig. 1(c) shows optical and scanning electron microscope (SEM) images of a fabricated disk array and a selected single disk. The images attest the well-defined circular boundary and flat top of the disk. Also the SEM cross section shows continuously co-etched sidewalls, critical for ensuring high Q cavity modes. 
(a)

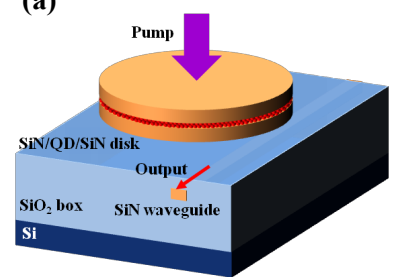

(b)

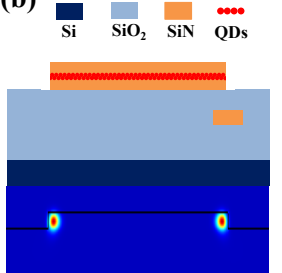

(c)

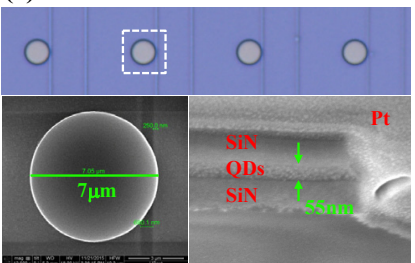

Fig. 1. (a) Schematics of the device configuration. (b) Cross-sectional schematics of the device (upper) and the simulated field intensity profile of the fundamental TE WGM mode (lower). (c) Optical and SEM images of fabricated devices. Top panel: an array of disks on a chip. Bottom panel: top-view of $7 \mu \mathrm{m} \mathrm{SiN} \mathrm{disk} \mathrm{coupled} \mathrm{to} \mathrm{the} \mathrm{buried} \mathrm{waveguide} \mathrm{and} \mathrm{cross} \mathrm{section} \mathrm{of} \mathrm{SiN/QD/SiN} \mathrm{layers} \mathrm{made} \mathrm{by} \mathrm{focused} \mathrm{ion-beam} \mathrm{milling.}$
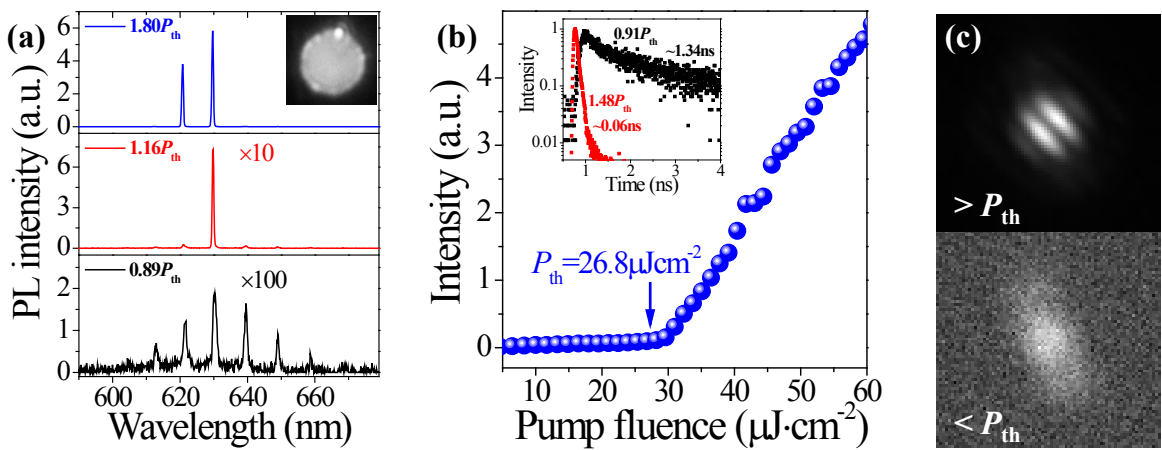

Fig. 2. (a) PL spectra of the $7 \mu$ m-diameter disk under different pump fluences. Top inset: PL image of the lasing disk. (b) Integrated PL intensity as a function of pump fluence. Inset: time-resolved PL decay traces at different pump power. (c) Interference patterns below and above threshold.

\section{Laser Characterization}

The devices were characterized on a micro-photoluminescence ( $\mu$-PL) set-up using a $400 \mathrm{~nm}$ pulsed laser with few picoseconds duration and an almost flat-top profile. PL signals are collected from the cleaved waveguide facet in an optical fiber and analyzed by a high-resolution spectrometer. Fig. 2(a) shows the PL spectra of a $7 \mu \mathrm{m}$-diameter disk with a lasing threshold fluence of $P_{\text {th }}=26.8 \mu \mathrm{J} \cdot \mathrm{cm}^{-2}$. Below threshold, the spectrum exhibits typical WGM modes in the disk within the envelope of the broadband spontaneous emission of the QDs. The extracted Q factor after subtraction of QD absorption is $\sim 2,000$ at $\sim 630 \mathrm{~nm}$ for the fundamental TE WGM, equivalent to a loss of $78 \mathrm{~cm}^{-1}$. In a waveguide with the same thickness of QDs, we measured an optical net gain of 100-120 $\mathrm{cm}^{-1}$, exceeding this loss. With increasing the pump fluence to $1.16 \cdot P_{\mathrm{th}}$, a $\sim 40$ times increase of the intensity is observed simultaneously accompanied by a narrowing line-width from $0.58 \mathrm{~nm}$ to $0.14 \mathrm{~nm}$ for the WGM at $629.43 \mathrm{~nm}$. When further increasing the pump intensity, a second lasing mode appears at shorter wavelength $(\sim 620.48 \mathrm{~nm})$. The lasing is concomitant with the emergence of scattered light of the WGM mode in the PL image of the disk. We plot the integrated PL intensity versus the pump fluence in Fig. 2(b), clearly showing the lasing threshold. The time-resolved PL reveals a significantly reduced lifetime from the spontaneous (ns) to stimulated emission regime (ps). Further measurement with high-resolution streak camera confirms the stimulated emission lifetime of 3-6 ps. The temporal coherence is characterized by means of a Michelson interferometer. Fig. 2(c) shows the recorded interference patterns from the waveguide facet below and above threshold. The sharp change of the visibility indicates a distinct enhancement of coherence. We measured a coherence time of 0.29 ps and 3 ps below and above threshold respectively with the latter mainly determined by the stimulated process within the pulsed excitation scheme.

\section{Conclusion}

In summary, we have demonstrated for the first time on-chip integrated SiN microlasers with low thresholds based on QDs. Our results represent a significant step toward the development of integrated light sources on SiN photonics platform using QDs as broad and tunable optical gain materials and thus open up new paths to a wide range of applications.

\section{References}

[1] V. I. Klimov et al., "Optical gain and stimulated emission in nanocrystal quantum dots," Science 290, 314(2000).

[2] C. Dang et al., "Red, green and blue lasing enabled by single-exciton gain in colloidal quantum dot films,” Nat. Nanotechnol. 7, 335(2012).

[3] W. Q. Xie et al., "Fabrication and characterization of on-chip silicon nitride microdisk integrated with colloidal quantum dots," Opt. Express 24, A114 (2016).

[4] W. Q. Xie et al., "Low-loss silicon nitride waveguide hybridly integrated with colloidal quantum dots," Opt. Express 23, 12152(2015).

[5] M. Cirillo et al., ""Flash" Synthesis of CdSe/CdS Core-Shell Quantum Dots,” Chem. Mater. 26, 1154(2014). 